\title{
Novel trends in the assembly process as the results of human - the industrial robot collaboration
}

\author{
Radovan Holubek ${ }^{1, *}$, Roman Ružarovský ${ }^{1}$, Daynier Rolando Delgado Sobrino ${ }^{1}$, Peter \\ Koštál ${ }^{1}$, Adam Švorc ${ }^{1}$ and Karol Velíšek ${ }^{1}$ \\ ${ }^{1}$ Institute of Production Technologies, Faculty of Materials Science and Technology in Trnava, \\ Slovak University of Technology in Bratislava, Bottova 25, 91701 Trnava, Slovakia
}

\begin{abstract}
The contribution is focused on the creation of an idea proposal and simulation of the assembly system in cooperation of the human and the industrial robot. The aim of the research is to verify the feasibility of this cooperation between the human and the industrial robot on the basis of the created simulation in the assembly process. The important step of the design this collaboration is the determination of rules and safety of this cooperation. The paper also presents the method of working with the selected software and its functionalities and sequence of steps at the simulation creation. The objective of the research is the evaluation of the idea proposal of the collaborative assembly system on the basis of the created simulation. The analysis and evaluation of the simulation confirm the feasibility and safety of the cooperation of the man and robot and also verified the possibility of assembly made by man and robot from the disposition and dimension on point of view of the proposed workplace.
\end{abstract}

\section{Introduction}

Currently, it can be concluded that intelligent human and robot collaboration takes place in the foreground. This is mainly due to increasingly complex customer requirements, as well as efforts to gain the highest market competitiveness. The implementation of collaborative workplaces has its justification for shortening innovation cycles and a more flexible response to the more predictable market demands. In processes such as human with fast moving robot cooperation, it is necessary to take extra protection of persons [1].

The role of intelligent human cooperation with the robot can be considered to facilitate the recurrent, dangerous, and often very demanding work activities [2, 3]. The standard role of the human in the manufacturing working is separated from the industrial robot. The reason is preventing collisions and situations that directly threaten human health in the production process. The concept of human-robot collaboration can be seen as a common work in which these two action members complement in the manipulation, palletizing, surface treatment, assembly [4], disassembly [5] and other technological operations.

* Corresponding author: radovan.holubek@stuba.sk 


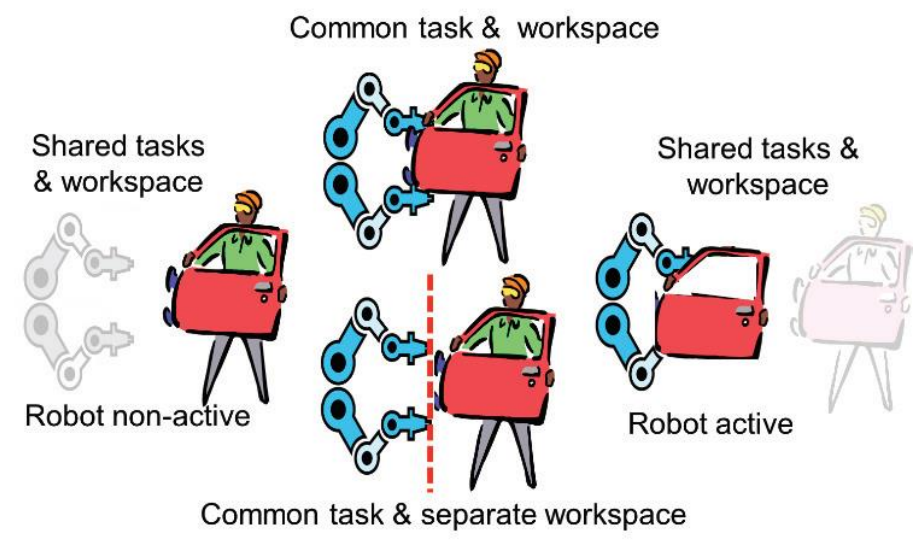

Fig. 1. Taxonomy of Human-Robot collaborative tasks and workspaces.

The human operators and robots are able to perform either single or cooperative tasks. The robot and the human operator could have a common task and workspace, a shared task and workspace, or a common task and a separate workspace (Fig. 1) [6].

The collaborative robot is also usable in control and tuning applications where its use is advantageous because it eliminates the potential failure of human factor. The robot is able to position itself in harder places, but at the same time it can achieve better accuracy even at higher speeds of movement. It is very advantageous to point out the fact that man can be exempted from monotonous activities and can devote himself to more valuable tasks and thought processes. The advantage of collaborative, also called cooperating workplaces is the fact that they combine the advantages of human's manual work and a fully automated workplace with an industrial robot [7]. The aim of the contribution is to verify the feasibility of this collaborative-collaboration based on the simulation of the conceptual design of the human-robot assembly workplace.

\section{Objectives}

The work deals with the idea of the assembly process, in which human will cooperate with a collaborative robot. The assembly process in the proposed collaborative workplace is simulated in the Siemens Tecnomatix Process Simulate software. An industrial robot performs predetermined tasks throughout the manufacturing process. The robot is capable of handling with adequate accuracy with hot, heavy but also sharp objects of manipulation. Compared to humans, however, it is not flexible in tasks in more complicated assembly processes. Therefore, if a human and a robot cooperate, it can also perform more complex tasks that would not be possible without their cooperation. Care must be taken to ensure its safety and health in operations where the working robot is near a human. These new safety standards have been defined by the American Association RIA - Robotic Industries Association in 2013 and have been approved by the US National Institute, designated ANSI / RIA R15.06-2012. This standard has been harmonized with the International Standard for Safety of Robots ISO 10218 [8]. Among the leading manufacturers of collaborative robots are the following companies: Universal Robots (UR3, UR5 and UR10), Rethink Robotics (Baxter, Sawyer), KUKA (LBR iiwa), ABB (Yumi, Roberta), F\&P Robotics (P-Rob), Fanuc (CR-35i). 


\section{Methods}

The research method is focused on the evaluation of idea design of a collaborative assembly workplace based on the simulation. Simulation allows to come to analyse the process itself before putting the real production system into operation and debug any errors or collisions [9-11]. The basis of the designed assembly workplace is the human - industrial robot cooperation. It was considered the subject of assembly in which we could connect the benefits of human work (such as the ability to manipulate in assembly) with the benefits of robot work (e.g. use of precision and repeatability). The simulation analysis will verify or our claim about the effectiveness of the collaboration is true or not. The first stage of the design was to determine the subject of the assembly.

\subsection{Design of a collaborative assembly workplace}

The first stage was mainly in the plane of thought processes, inspiration was sought in model-like, assembled workplaces. The second stage included the transfer of design from the idea plane to the software modelling environment and to give it the appropriate dimensions and shapes. Here it was adapted or modified the ideological proposals (retaining the essence of the idea proposal). It was achieved that the conceptual design of the cooperation workplace would be the most feasible.

The subject of the assembly is the engine block on which the robot applies the sealant, and then the man puts the lid on the engine block, assembles and screws the screws of the motor cover. The main idea of the assembly workplace is the industrial robot cooperated with the human operator. It is used the cooperative industrial robot from Universal Robots Company, the type UR5, Fig. 2. It was considering compliance with safety standard 10218, which specifies the rules of the collaborative workplace.

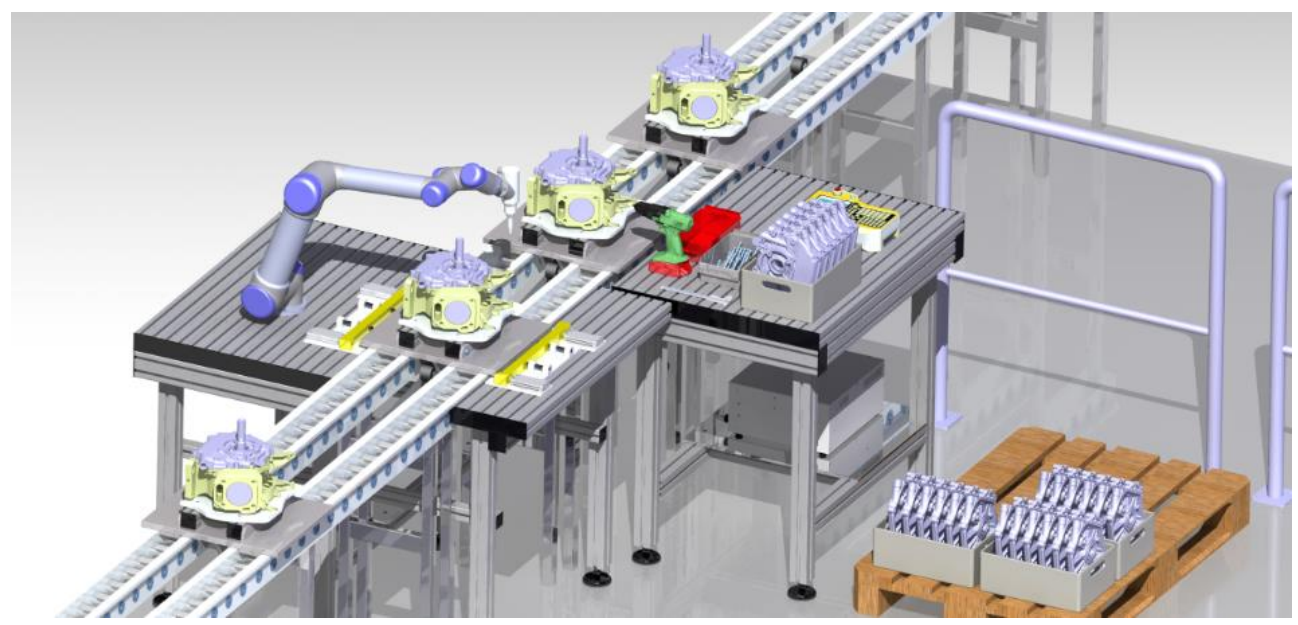

Fig. 2. Designed collaborative assembly workplace with robot UR5.

\subsection{The simulation of the assembly process with human - the industrial robot collaboration}

In this part of the paper is briefly described the creation of the simulation sequence. The creation of the simulation was actually a composition into a coherent time sequence with the logical continuity of the activities of the already created primary operations of 
individual elements and humans. The simulation has a duration of 150 seconds, starting with the movement of the carrier plate with the engine block from the starting point to the conveyor and ending at the end point at the end of the conveyor by assembling the entire engine block, the lid and the screw seal. The simulation course was divided into nine basic steps:

1. A delivery of the carrier plate with a motor block on the conveyor,

2. Carrier plate with engine block stopped by a stopper, set and clamped by a clamp, while man is coming to his workplace and the robot moves into the preparatory position, Fig. 3a.

3. The industrial robot is applying a sealant (Loctite 510), at the same time the human operator has taken over and holds the lid and controls the activity performed by the robot. Fig. 3b.

4. The robot UR 5 leaves the workspace after completing his activities, and when he leaves the working position, the human operator places the lid on the engine block (we've got enough screws with screws to prevent the screws from falling out of the lid), Fig. 3c.

5. The robot has reached the Home position, at the same time the human operator has taken the screwdriver and performs the action of screw-drivers on the motor lid, Fig.3d.

6. The human has complete the screwing operation and he has put the screwdriver on the defined place of storage on the side of the assembly desk.

7. The human moves to the designated point where he check a conveyance and at the same time is given the instruction for opening the support plate clamp with the assembled motor block.

8. The leaving of the carrier plate with a motor block on the conveyor and the preparing for the next operation.

9. Repeat the assembly process.

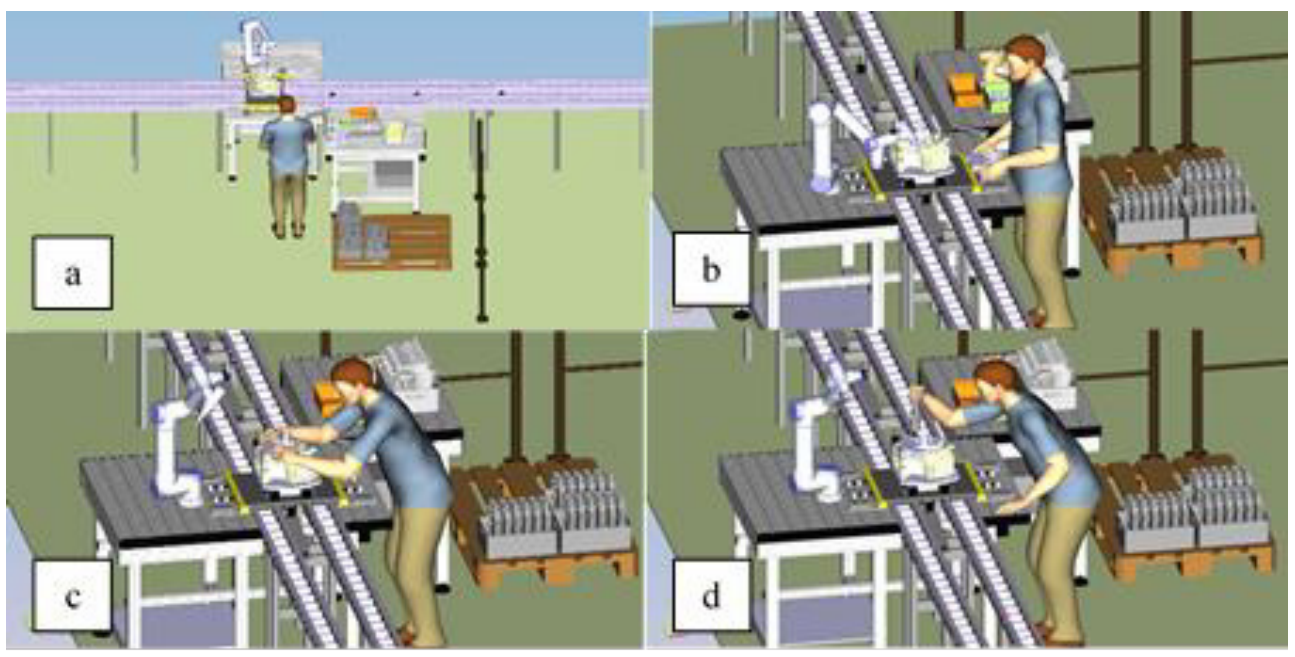

Fig. 3. Designed collaborative assembly workplace with robot UR5 and human operator.

\section{Results from the simulation}

The design of the assembly workplace was based on the idea of a hand-assembled workstation with a low level of automation. We have three goals in the proposal: 
1. Human being the manipulation of less effort to accuracy, but also complicated with regard to the shape of the lid (we are considering several of its types).

2. Remove the human activities straining for its precision and concentricity (putting a seal around the perimeter of the engine block) and industrial cooperate robot being work (UR 5).

3. To preserve the original workspace (space efficiency, costs) while at the same time achieving the safety of the workplace in which these two members will work on a common subject.

The final point is obvious and necessary at the same time, the use of an industrial robot designed to work with a human. The workplace is not protected by fencing, optical gates or other systems. Human safety is only maintained by using a UR 5 robot that meets the conditions of collaboration.

The purpose of the simulation was to verify and evaluate the proposed dimensions of the workplace elements, its layout solution, With respect to the positioning of the robot with the effector and the necessary human availability in the assembly positions while maintaining ergonomic conditions. Based on observations during the simulation, it would like to give us found the design in which we in the subsequent correction made changes: changing the method of fixing and clamping the engine block onto the carrier plate, changing the method of clamping the support plate during assembly or to find and choose a faster way of mounting and clamping the support plate during assembly, by observing the movements and positions of the human operator when he screwing, it would be convenient to place the engine block at a lower height, Use the battery-powered screwdriver (or pneumatic actuator) on the balancer to reduce screwing stress, The end effector for the application of the seal is designed so as to meet the safety of the collaborative workplace (its surface is coated with softened PVC, like the robot surface).

The following figure, Fig. 4, it can be observed the robot and human workspace. It was verified that the robot does not interfere with a zone where a person is waiting to fit the lid on the engine block.

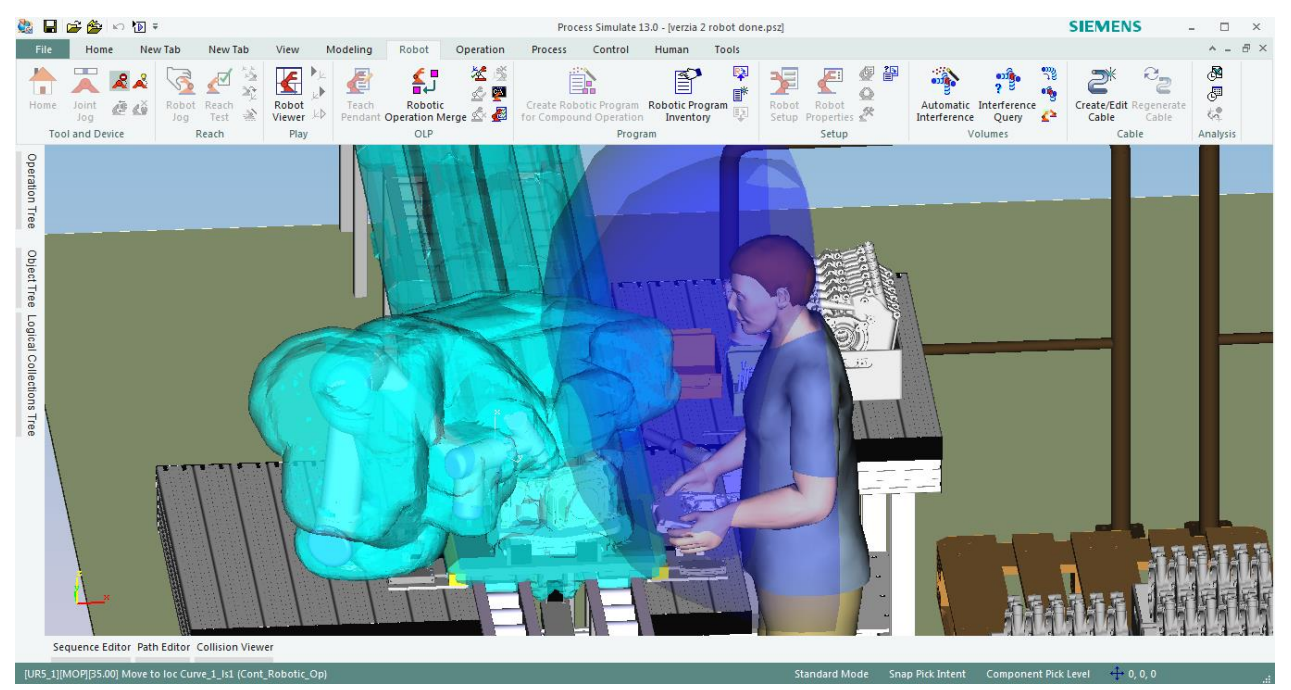

Fig. 4. The robot and human workspace, the Swept Volume function in Process Simulate. 


\section{Conclusion}

Nowadays it can be seen growing interest and also the increasing activity in the field of innovation and innovative approach in different directions their competence, especially in Industrial Revolution in Automation - Industry 4.0. The human - robot cooperation definitely belong to this sphere. The aim was to create a simulation of the assembly process in the proposed workplace to verify the possibility of human interaction with the robot while preserving the required security. Followed it was analysed and evaluated the idea design of our workplace, it was mentioned the areas in which should be modified it and were made the additional corrections. The main contribution of this paper is the fact verified by the simulation that collaboration of a human with a collaborative robot in the common space is feasible.

This research was supported by the STU Grant "Scheme for Support of Excellent Teams of Young Researchers - (1349 - Use of simulation methods in the context of the Virtual Commissioning as a tool for production systems in the Digital Factory).

\section{References}

1. J. Teiwes, T. Bänziger, A. Kunz, K. Wegener, ICAC (2016)

2. D. Antonelli, S. Astanin, Proc. CIRP, 41, 352 (2016)

3. D. Schröter, P. Jaschewski, B. Kuhrke, A. Verl, Proc. CIRP, 55, 2016, 12 (2016)

4. Å. Fast-Berglund, F. Palmkvist, P. Nyqvist, S. Ekered, M. Åkerman, Proc. CIRP, 44, 175 (2016)

5. H. Cheng, W. Xu, Q. Ai, Q. Liu, Z. Zhou, D. Truong Pham, Proc. Man., 10, 26 (2017)

6. G. Michalos, S. Makris, P. Tsarouchi, T. Guasch, D. Kontovrakis, G. Chryssolouris, , Proc. CIRP, 37, 248 (2015)

7. P. Tsarouchi, A.-S. Matthaiakis, S. Makris, G. Chryssolouris, Int. Jour. of Comp. Int. Manuf., 30, 6 (2017)

8. Ch. Vavra, Control Engineering Cz, 11(4-83), 12 (2016)

9. K. Velíšek, R. Holubek, D. R. Delgado Sobrino, R. Ružarovský, N. Vetríková, MATEC Web of Conf. (2017)

10. K. Baizid, A. Meddahi, A. Yousnadj, S. Ćuković, R. Chellali, MATEC Web of Conf. (2016)

11. Z. Tuma, L. Kotek, J. Tuma, F. Bradac, MM Sci. Jour., 1, 1, 1487 (2016) 